\title{
My opinion: obesity interventions need integration across sectors and translation of good research to communities
}

\section{Opinion}

Obesity is a global problem Global Nutrition Report 2016 indicate an increasing trend. ${ }^{1}$ More than half $(52.0 \%)$ of adults 18 years and above worldwide are either obese or overweight. ${ }^{2}$ Similarly, obese children have increased globally with Africa and Asia recording up to double the number of obese/overweight children under 5years between 1990 and $2014 .^{2}$

The consequences of these increasing prevalence's of obesity is directly the increase in body mass index, increased risk for several non-communicable diseases, ${ }^{3}$ increased disability and premature death in adulthood for children. ${ }^{2}$ Obesity related deaths have increased compared to underweight related deaths. A more indirect effect is increased health care cost, ${ }^{1}$ reduced workforce and development in nations. This is an indication that obesity greatly affects the individual and affects families, communities or nations. Obesity, a threat to life and the growth of populations have inspired more research, ${ }^{4}$ and innovation. Deservedly, great advances have been made in generating evidence in nutrition education programming, medical procedures, medications and policies to directly reduce or create an enabling environment ${ }^{5}$ to reduce obesity.

Advances in nutrition research, technology and findings need to be translated into real life situations. Many reviews and metaanalyses on obesity related interventions have postulated ideas on mice and human studies. Mindful of the need for ethical research, many findings such as the role of Omega- 3 fatty acids ${ }^{6}$ in reducing obesity requires practicalisation. Many of these findings appear only in publications and have not been scaled up to human studies or have not been tested in free living situations. Thus, a need for more funding and partnerships in translation research.

The great deficiency in our efforts as concerned and health care workers has been the struggle for credit and credibility of research or program approaches. This has led to the implementation of parallel interventions. The bench, clinic and community researchers/ programmers' efforts are united in their single goal to reduce obesity and its consequences. This we need to showcase in integration with education messages. Obesity is mainly a nutrition-related health condition, but also a social condition. ${ }^{7}$ The recipients of education/ treatment need to integrate experts' advice on food, exercise and emotional management, but mostly lack the tools to do so efficiently. However, they are not alone, the experts lack these tools too. We need a united front, relating messages which build on the strengths of each other.

Sustained behavior change in an enabling environment is key. Healthier, affordable food choices should be available and accessible to all people. Similarly, it can be said about the treatment for already obese adults and children and preventive initiatives. Sustainability of
Volume 5 Issue 2 - 2016

Brenda AZ Abu
Nutritional Sciences Department, Texas Tech University, USA

Correspondence: Brenda AZ Abu, PhD, Nutritional Sciences Department, Texas Tech University, College of Human Sciences, Texas, USA. Email brenda.a.abu@ttu.edu

Received: August 19,2016 | Published: October 08, 2016

interventions needs a discussion among research, policy, program and funding stakeholders to drive more integrated and multi-disciplinary approaches.

Obesity rates are increasing globally leading to great effects on individuals and nations. Health bearers have made tremendous progress in identify, treating and preventing the increase in obesity prevalence rates. However, the translation of good research beyond publications, intervention integrated and multidisciplinary approaches are important to lead to sustained progress in addressing obesity.

\section{Acknowledgements}

None.

\section{Conflict of interest}

The author declares no conflict of interest.

\section{References}

1. Global Nutrition Report 2016: From Promise to Impact: Ending Malnutrition by 2030. Washington DC: International Food Policy Research Institute (IFPRI).

2. World Health Organization (WHO). Obesity and overweight. Fact sheet. 2016.

3. World Health Organization (WHO). Global Action Plan For the Prevention and Control Of Non-Communicable Diseases 2013-2020. Switzerland; 2016.

4. Eric Stice, Heather Shaw, Nathan Marti C. A Meta-Analytic Review of Obesity Prevention Programs for Children and Adolescents: The Skinny on Interventions that Work. Psychol Bull. 2006;132(5): 667-691.

5. World Health Organization (WHO). Commission on Ending Childhood Obesity. Switzerland; 2016.

6. Buckley DJ, Howe PR. Long-Chain Omega-3 Polyunsaturated Fatty Acids May Be Beneficial for Reducing Obesity-a Review. Nutrients. 2010;2(12):1212-1230

7. Parsons TJ, Power C, Logan S, et al. Childhood predictors of adult obesity: a systematic review. International Journal of Obesity. 1999;23(Suppl 8):S1-S107. 